\title{
Review of: "DIAGNOSTIC ACCURACY OF BONE SCINTIGRAPHY IN THE EARLY PREDICTION OF MRONJ"
}

\section{Christian Bacci ${ }^{1}$}

1 University of Padua

Potential competing interests: The author(s) declared that no potential competing interests exist.

Interesting tips to our clinical practice. 